\title{
Transições dos jovens para o mercado de trabalho, primeiro filho e saída da escola: o caso brasileiro
}

\author{
Elzira Lúcia de Oliveira* \\ Eduardo Gonçalves Rios-Neto** \\ Ana Maria Hermeto Camilo de Oliveira***
}

\begin{abstract}
Este artigo investiga o relacionamento entre as idades em que ocorrem as transições de saída da escola, entrada no mercado de trabalho e formação da família. A metodologia adotada foi a análise de sobrevivência. Realizou-se análise descritiva, por meio de modelo não paramétrico (Kaplan Meier), para estimar funções de sobrevivência para cada uma dessas transições por sexo. Foi também feita abordagem semiparamétrica (Modelo de Riscos Proporcionais de Cox), incluindo-se variáveis como educação da mãe e do pai, situação de residência até os 15 anos, etc. A base de dados utilizada neste artigo foi a Pesquisa sobre Padrões de Vida - PPV. Os resultados captaram a experiência de transição das pessoas com 20 a 49 anos na época da pesquisa (1996-1997) e, portanto, não necessariamente traduzem a experiência dos jovens que estão realizando as transições nessa década. A importância dos achados do estudo está justamente nessa característica, pois, se para a experiência dessas coortes fica evidente uma seqüência de transições e a idade ao primeiro filho reduz em apenas 0,9\% o risco de deixar a escola, é porque a transição para o primeiro filho não representava o principal motivo de evasão escolar. Assim, os desafios identificados, as idades e os fatores intervenientes nas transições são informações de grande relevância para políticas públicas de educação, qualificação, geração de emprego, habitacionais e de saúde.
\end{abstract}

Palavras-chave: Transições de jovens. Mercado de trabalho. Análise de sobrevivência.

\section{Introdução}

A literatura sociológica defende que o processo de transição para a maioridade envolve quatro eventos principais: sair de forma definitiva da escola; encontrar um trabalho temporário ou permanente por meio do qual o indivíduo possa suprir a si ou a uma possível família dos recursos materiais necessários à sobrevivência; formar a primeira união relativamente estável, ou seja, viver com o companheiro em uma mesma residência; e, por fim, ter o primeiro filho (MODELL FURSTENBERG e

\footnotetext{
*Professora da Universidade Candido Mendes - Campos. Mestrado em Planejamento Regional e Gestão de Cidades. Doutorado em Demografia pelo Cedeplar/UFMG.

** Professor da Universidade Federal de Minas Gerais - Cedeplar. PhD em Demografia Econômica pela University of California at Berkeley, EUA.

**^ Professora da Universidade Federal de Minas Gerais - Cedeplar. Doutora em Demografia pelo Cedeplar/UFMG.
} 
HERSHBERG, 1976; CAVALLI e GALLAND, 1993; IEDEMA, BECKER e SANDERS, 1997; SHANAHAN, 2000).

A transição para a maioridade é um processo que envolve diversas etapas experimentadas ao longo do tempo. Assim, podem-se observar indivíduos em vários estágios da transição. Nas diversas sociedades, há pessoas que jamais completarão todo o processo, o que, no entanto, não significa que elas não atingiram a maioridade. À medida que o indivíduo assume papéis sociais destinados aos adultos, seja na estratificação ocupacional, seja no matrimônio ou nas tarefas da paternidade, isso quer dizer que ele está trocando as atribuições sociais da juventude por aquelas destinadas à maioridade (SHIZZEROTTO e LUCCHINI, 2002).

A literatura econômica no Brasil e na América Latina tem se preocupado, basicamente, com a transição que envolve a saída da escola e a entrada no mercado de trabalho, ignorando a formação de família compreendida como a primeira união ou o nascimento do primeiro filho - enquanto importante processo intermediário ou simultâneo à entrada no mercado de trabalho e à transição para fora da escola.

O aumento do nível de escolaridade e da permanência na escola (independentemente do nível de escolaridade) induz à simultaneidade entre as atividades escolares e as do mercado de trabalho. O papel da formação de família como um processo simultâneo aos demais pode, inclusive, evidenciar diferenças significativas de gênero. Enquanto escola e trabalho podem ser mais importantes para os homens, as mulheres têm maior preocupação com a escola e a maternidade.

Este artigo explora as transições principais - entrada no mercado de trabalho, saída da escola e ter o primeiro filho - sem, no entanto, se preocupar com a proporção dos indivíduos que completaram ou não todo o processo de transição. O objetivo é entender em que medida, no Brasil, existe relação entre as idades nas quais ocorrem tais processos, ou seja, pretende-se identificar um padrão etário e seqüencial de transição, baseado na experiência dos indivíduos que forneceram informações à Pesquisa sobre Padrões de Vida - PPV, levada a campo em 1996 e 1997.

\section{Antecedentes}

O marco conceitual desse tipo de investigação remete a três linhas de estudos. A primeira refere-se aos tradicionais relacionamentos entre escola e trabalho, que constituem o foco principal de economistas e demógrafos brasileiros. A segunda explora as características domiciliares e individuais determinantes das transições: entrada no mercado de trabalho, formação de família e saída da escola.

Seguindo essas duas linhas de investigação, alguns trabalhos brasileiros analisam como os jovens alocam o tempo entre as atividades de trabalho e estudo: uma ou outra exclusivamente, ambas simultaneamente ou nem uma nem outra. $O$ que se procura são evidências sobre os determinantes dessas escolhas. Os estudos nessa linha revelam que as características sociodemográficas da família são importantes, ressaltando, ainda, a importância intergeracional da educação, uma vez que maior escolaridade dos pais determina em grande parte a escolha do jovem pela escola (CORSEIL, SANTOS e FOGUEL, 2001; LEME e WAJNMAN, 2000).

Finalmente, a terceira analisa a relação causal entre os eventos, ou seja, em que medida a permanência na escola atrasa a primeira união, ou ainda em que proporção a primeira união acelera a saída da escola. Também são exploradas as relações causais entre entrar no mercado de trabalho e sair da escola, bem como a associação entre as transições simultâneas: se a entrada no mercado de trabalho acionaria o processo da primeira união e a saída da escola; ou se a primeira união aceleraria a transição da escola para o mercado de trabalho. A literatura chama a atenção para a existência de características não observáveis que afetariam todos os processos, o que demanda tratamento específico para obtenção de resultados confiáveis e livres de viés. Ressalta-se, também, a hipótese de que a entrada na união ou no mercado 
de trabalho, sem completar a formação escolar, influenciaria o estoque de capital humano individual, o que prejudicaria os ganhos futuros por desvantagens tanto no mercado de trabalho quanto no mercado de casamento.

$\mathrm{Na}$ literatura latino-americana, destacase o trabalho de Stupp e Cáceres (2001), que realizam um minucioso exame da relação entre a idade ao ter o primeiro filho e a escolaridade da mulher, considerando a idade de conclusão da educação em El Salvador. O risco relativo de ter o primeiro filho foi analisado segundo diversas características da mulher, por meio do Modelo de Riscos Proporcionais de Cox. O principal achado foi que a idade com a qual a mulher sai da escola, e não o mais alto nível de educação alcançado, é um aspecto crítico do acompanhamento escolar feminino e afeta o risco de ter um filho.

$\mathrm{Na}$ literatura européia, entre os estudos que relacionam características individuais e domiciliares, destaca-se Coppola (2001), que investiga o relacionamento entre a formação de família e as transições para o emprego na Espanha e na Itália. Na Espanha, a mulher apresenta a mesma relação entre união e participação na força de trabalho, embora mais fraca em comparação aos homens. Os resultados encontrados para a mulher italiana sugerem que a entrada na primeira união e a entrada na força de trabalho podem não ter qualquer efeito forte uma sobre a outra, devido à heterogeneidade não observada.

Outra forte relação de simultaneidade constatada encontra-se no tempo de permanência na escola e a idade à primeira união. Verificou-se que estes dois indicadores têm aumentando significativamente na Espanha e na Itália. Coppola e Aassve (2003) procuraram mostrar evidências empíricas da endogeneidade desses dois processos. Por um lado, a saída do sistema educacional aumenta o risco de entrar na primeira união, mas, por outro, existem características individuais não observadas que influenciam, simultaneamente, a ocorrência dos dois processos. Se o modelo não é controlado por essas características, os efeitos da conclusão da educação sobre a formação de família produzem um viés ascendente.

A seqüência desses eventos é também relacionada a normas sociais que variam de país para país. Assim, Espanha e Itália têm sido consideradas as últimas duas nações que apresentam padrão de saída dos jovens da casa dos pais com alta sincronização com a entrada na primeira união.

Diferente do norte da Europa, onde os laços de família são fracos, Itália e Espanha são protótipos do padrão mediterrâneo de transição da casa dos pais para o processo de formação de família, por manterem laços familiares conservadores. Por essa razão, tornam-se, recorrentemente, objetos de estudo do padrão dessas transições (BILLARI et al., 2000). Estes autores analisaram o padrão de saída da casa dos pais com a probabilidade de múltiplos destinos. A carreira profissional e educacional e o último grau de escolaridade concluído foram inseridos como determinantes desses múltiplos destinos: transição para uma residência autônoma, transição por motivo de casamento, transição para casa dos pais de um dos cônjuges (patrilocal unions), transição para coabitação e, ainda, o papel da maternidade precoce.

Concluiu-se que o fato de estar na condição de estudante impede a transição para a formação de família (seja morando na casa dos pais ou não, seja para união consensual ou marital), entretanto, pode promover a transição para um domicílio autônomo. A exceção fica por conta das muIheres espanholas, para as quais o fato de permanecer na escola impede o casamento, mas não a coabitação, atuando positivamente na transição para a autonomia. Na Espanha, a coabitação apresenta correlação com níveis educacionais mais elevados, ao passo que, na Itália, não se verifica seletividade com o nível educacional.

O papel desempenhado pela carreira profissional confirma um viés por gênero já previsto na literatura, ou seja, possuir um trabalho aumenta significativamente as chances dos homens de transitarem para o casamento. Já para as mulheres, verificou- 
se uma associação negativa, embora o fato de ter uma vasta experiência profissional e ter tido um trabalho aumente as chances de transição. Afora as diferenças de gênero observadas, ter um trabalho, incoerentemente, diminui as chances de transição para a autonomia, possivelmente pela incerteza quanto à estabilidade do emprego.

A literatura européia ainda explora os fatores que influenciam o padrão de transição em alguns países. Nesse sentido, Shizzerotto e Lucchini (2002) analisam a ordem cronológica das idades nas quais se completa cada etapa, o tempo decorrido entre a etapa subseqüente e a duração de todo o processo de transição. Os recursos materiais disponíveis, aparentemente, condicionam a velocidade da maioria das transições individuais para a maioridade. Como conseqüência, a situação econômica do país, a dinâmica do mercado de trabalho, a generosidade e o grau de universalização do regime de bem-estar afetam a trajetória de transição. Entretanto, a idade, a duração e a seqüência pressupõem que o papel social designado ao adulto é afetado pelo arranjo institucional, cultural e processo histórico do país.

Acrescenta-se à literatura elencada o trabalho desenvolvido por Biddecom e Bakilana (2003), que investigam o tempo, a densidade e a seqüência das transições realizadas por adolescentes e jovens adultos, de ambos os sexos, para a atividade sexual, gravidez, uniões e saída da escola na África do Sul. Além da densidade, a seqüência dos eventos também merece investigação, pois a duração de um evento pode determinar o início e a duração de outro evento, ou mesmo a probabilidade de acionar outro evento. Os resultados não apresentaram alta densidade de transição no período da adolescência até os vinte anos. Além disso, foram verificadas importantes diferenças entre os grupos populacionais no que diz respeito às transições estudadas, bem como uma grande desordem em termos da variedade de combinações e seqüências cronológicas das transições.

\section{Dados e metodologia}

\section{Fonte de dados}

A PPV foi aplicada no Brasil nas Regiões Nordeste e Sudeste, pelo Instituto Brasileiro de Geografia e Estatística - IBGE e pelo Banco Mundial. No Nordeste foram investigadas amostras nas regiões metropolitanas de Fortaleza, Recife e Salvador, restante da área rural e restante da área urbana. Na Região Sudeste, os estratos considerados foram as regiões metropolitanas de Belo Horizonte, Rio de Janeiro e São Paulo, restante da área urbana e restante da área rural.

O tamanho da amostra para cada estrato geográfico foi fixado em 480 domicílios, sendo estipulados 60 setores, em cada estrato geográfico, e oito domicílios, em cada setor.

O questionário foi planejado para fornecer um conjunto de informações integradas com o objetivo de entender os diversos aspectos de distribuição do bem-estar e pobreza. Além disso, investigaram-se, retrospectivamente, o histórico de educação e a atividade econômica de homens e mulheres e também a história de fecundidade das mulheres de 15 a 49 anos na data da pesquisa. Com isso, obtêm-se dados retrospectivos a respeito das idades com as quais os indivíduos experimentaram os eventos investigados neste trabalho.

\section{Metodologia}

Neste trabalho, adotou-se a análise de sobrevivência como estratégia de verificação empírica do problema tratado. Em uma seção mais descritiva, utilizar-se-á análise não paramétrica para obter curvas de sobrevivência em cada um dos eventos investigados. Posteriormente, serão incluídas covariáveis no modelo, para o que se adotará o modelo semiparamétrico de riscos proporcionais de Cox.

\section{Análise não paramétrica}

Para os propósitos deste trabalho, serão utilizados os métodos da função de sobre-vivência não paramétrica (KaplanMeier) e função de risco (Hazard). A análise de sobrevivência descreve diretamente a 
experiência de sobrevivência da coorte em estudo, enquanto a função de risco informa sobre a probabilidade da ocorrência do evento investigado condicionada à sobrevivência no tempo $t$.

Os métodos discutidos consensualmente na literatura (ALLISON, 1995; KLEIN e MOESCHBERGER, 1997; RETHERFORD e CHOE, 1993, entre outros) usam dados que lidam com o tempo até a ocorrência de qualquer evento bem definido (duração). Nesse caso, a variável de interesse é a idade, em anos, da ocorrência do primeiro filho, da saída da escola e do primeiro trabalho. Como os eventos estão distribuídos ao longo de um tempo determinado, a origem da escala de tempo é definida a partir do início da observação, que pode variar entre os indivíduos de um grupo em estudo. Quando se trata de dados retrospectivos, como neste estudo, a origem da escala de tempo é bem definida pela idade do indivíduo no evento que marca o início da contagem, ou seja, a idade a partir da qual se considera o início de cada evento.

No caso da entrada no mercado de trabalho, levou-se em conta a menor idade relatada pelos indivíduos que tinham entre 20 e 49 anos na época da entrevista. Para a saída da escola, a menor idade declarada pelos indivíduos que tinham entre 20 e 49 anos na época da entrevista marcou o início da contagem desse evento. E, para o nascimento do primeiro filho, considerou-se a menor idade mencionada pelas mulheres que tinham entre 20 e 49 anos na época da entrevista.

Optou-se pela experiência dos entrevistados de 20 a 49 anos para, em primeiro lugar, padronizar a amostra e tentar captar relações seqüenciais e de simultaneidade entre os eventos e, em segundo, minimizar problemas de memória, comuns em dados retrospectivos, bem como qualquer viés de seletividade causado pelos jovens em idade de transições.
Deve-se levar em conta a possibilidade de o evento não ocorrer durante o tempo de observação. Neste caso, a única razão é que existem pessoas no grupo de interesse que não haviam experimentado o evento até a data da entrevista, o que impossibilita conhecer o tempo de ocorrência até o evento. Entretanto, tais dados não devem ser ignorados, pois se sabe que o tempo até o evento é, no mínimo, algum valor. ${ }^{1}$ Um indivíduo será censurado à direita neste trabalho se, até o tempo $t$ (idade de ocorrência do evento investigado), ele não tiver experimentado algum dos eventos de interesse. Neste trabalho, não existe censura à esquerda, ${ }^{2}$ pois o tempo exato transcorrido até os eventos de interesse é conhecido.

A análise exploratória dos dados foi realizada por meio de tabelas de contingências, a fim de serem investigadas as relações de dependência entre os eventos. Primeiramente, fez-se uma breve introdução sobre as funções básicas da distribuição do tempo em análise de sobrevivência: a de distribuição da probabilidade; a de sobrevivência; a de densidade; e a de risco.

\section{A Função de distribuição da probabilidade}

O tempo de sobrevivência de um indivíduo, em algum tipo de análise de sobrevivência, é considerado uma variável aleatória T, que pode ser caracterizada por uma distribuição cumulativa chamada de distribuição de função.

$$
F(T)=\operatorname{Pr}[T<t], t \geq 0
$$

\section{Função de sobrevivência}

O método denominado Kaplan-Meier KM estima uma função de sobrevivência $\mathrm{S}(\mathrm{t})$ para os valores de $\mathrm{t}$ observados no banco de dados, na forma $\left(t_{i}, d_{i}\right), \ldots,\left(t_{n} d_{n}\right)$,

\footnotetext{
${ }^{1}$ Se uma mulher tinha 25 anos na data da pesquisa e ainda não havia tido o primeiro filho, conclui-se que, no mínimo, o tempo de sobrevivência para o evento em estudo é de dez anos. Essa mulher será considerada censura à direita.

${ }^{2} O$ tempo de sobrevivência também pode ser censurado à esquerda se tudo o que se sabe sobre o tempo até o evento é que ele é menor ou igual a algum valor. Muitos pesquisadores investigam meios de tratar dados censurados à esquerda e intervalo de censura. No entanto, a maior parte dos métodos de análise de sobrevivência consideram apenas os dados censurados à direita.
} 
sendo $d_{i}$ a situação de ocorrência do evento. Supõe-se que o t esteja ordenado assim: $t_{1}<t_{2} \ldots<t_{n}$. A cada evento ocorrido em $t_{i}$, $a$ probabilidade de sobreviver além de $t=t_{i}$ é reduzida pela multiplicação por $\left(r_{i}-1\right) / r_{i}$, em que $r_{i}$ é o número de indivíduos em risco exatamente antes do iésimo $t$.

Para algum valor não negativo de $t, F(t)$ é a probabilidade de o tempo de sobrevivência ser menor do que o tempo t. Descrevendo a variável aleatória $\mathrm{T}$, em termos da probabilidade de o tempo de sobrevivência ser no mínimo t, tem-se a Função de Sobrevivência $S(t)$, que é mais comumente usada em análise de sobrevivência:

$$
S(t)=1-F(t)=\operatorname{Pr}[T \geq t], t \geq 0
$$

\section{Função de risco (Hazard function)}

A função de risco é a taxa instantânea de a probabilidade de ocorrência do evento condicionada ao indivíduo não ter experimentado o evento ao tempo $t$.

$$
h(t)=\frac{f(t)}{S(t)} t \geq 0
$$

Como o risco ultrapassa a unidade, ele não pode ser pensado como uma probabilidade. Uma maneira de associar a função de risco ao tempo t, como uma probabilidade, é calcular a aproximação quando $\Delta \mathrm{t}$ tende a zero.

$$
h(t) \Delta t=\frac{F(t+\Delta t)-F(t)}{S(t)}
$$

O numerador da função é a probabilidade de o indivíduo em análise experimentar o evento entre o tempo t e t+Dt. Quando se divide por $\mathrm{S}(\mathrm{t})$, condiciona-se à sobrevivência no tempo t. Então, o risco no tempo t multiplicado por um pequeno incremento de tempo é uma aproximação da probabilidade de ocorrer o evento dentro desse pequeno intervalo de tempo após $t$, para o indivíduo que tenha sobrevivido ao tempo $t$.

\section{Análise semiparamétrica}

Quando se lida com grupos que apresentam características similares e se diferenciam apenas no tratamento sobre estudo, as respostas obtidas por meio da análise não paramétrica respondem satisfatoriamente à questão. Entretanto, quando não se tem idéia da homogeneidade entre os grupos e, ainda, se os grupos revelam características específicas do ponto de vista demográfico e comportamental que podem afetar o resultado, tais características podem ser usadas como covariáveis (variáveis explicativas) da variável resposta. Nesse caso, as covariáveis serão preditoras de fatores de risco para o evento, melhorando o grau de acurácia e reduzindo o viés do modelo não paramétrico que contempla apenas a comparação de funções de sobrevivência.

$O$ modelo que permite este tipo de análise, conforme citado em Alisson (2003), foi desenvolvido por Cox $(1972)^{3}$ e denomina-se Modelo de Riscos Proporcionais (Porportional Hazards Models).

Lembrando que T denota o tempo para ocorrência de um dado evento, baseado em uma amostra de tamanho n, este pode ser definido genericamente como se segue:

$$
\begin{aligned}
& T=f\left(t_{j}, \delta_{j}, \mathbf{Z}_{j}(t)\right) \mathrm{j}=1,2,3, \ldots, \mathrm{n} \\
& \text { onde: }
\end{aligned}
$$

$t_{i}$ : é tempo de observação do j-ésimo indivíduo;

$\delta_{j}$ : variável indicadora $\delta_{j}=1$ se evento tiver ocorrido e $\delta_{j}=0$ se o tempo de sobrevivência for censurado à direita;

$\mathbf{Z}_{j}(t)=\left(Z_{j 1}(t), \ldots, Z_{j p}(t)\right)^{t}$ é o vetor de covariáveis ou fatores de risco para o j-ésimo indivíduo no tempo t que poderia afetar a distribuição de T.

O vetor $Z_{j k}(t)$ 's, tal que $k=1,2,3, \ldots . p$, pode ser composto de variáveis que mudam no tempo, denominadas dependentes do tempo (time-dependent variable), como, por exemplo, diagnóstico atual, pressão

${ }^{3}$ COX, D. R. Regression models and life tables. Journal of the Royal Statistical Society, B 34, 187-220 (with discussion). 
sangüínea, situação ocupacional, matrícula em escola para indivíduos em idade escolar, entre outras. Esse vetor pode também ser composto por variáveis fixas, ou seja, já conhecidas no momento zero (sexo, grupocontrole, raça, diagnóstico inicial, situação ocupacional no começo da contagem do tempo, série em curso no princípio da contagem do tempo, entre outras). No caso específico deste trabalho, serão consideradas covariáveis de efeito fixo.

O modelo básico para função de risco no tempo $t$, para um indivíduo em risco, segundo o vetor $\mathbf{Z}$, é chamado de semiparamétrico porque apenas os efeitos das covariáveis são tratados parametricamente. A função de risco de referência é ajustada da forma não-paramétrica.

$$
h(t \mid \mathbf{Z})=h_{0}(t) c\left(\beta^{t} \mathbf{Z}\right)
$$

$h_{0}(t)$ : é uma função de risco de referência definida arbitrariamente;

$\boldsymbol{\beta}=\left(\beta_{1}, \beta_{2}, \beta_{3}, \ldots, \beta_{n}\right)$ : é o vetor de parâmetros das covariáveis;

$c\left(\beta^{t} Z\right)$ a função conhecida.

Como $h(t \mid Z)$ é obrigatoriamente positiva, um modelo comum para $c\left(\boldsymbol{\beta}^{\prime} \mathbf{Z}\right)$ é:

$$
c\left(\boldsymbol{\beta}^{t} \mathbf{Z}\right)=\exp \left(\boldsymbol{\beta}^{t} \mathbf{Z}\right)=\exp \left(\sum_{k=1}^{p} \beta_{k} Z_{k}\right)
$$

Assim, pode-se reescrever $h(t \mid \mathbf{Z})$ da seguinte forma:

$$
h(t \mid \mathbf{Z})=h_{0}(t) \exp \left(\boldsymbol{\beta}^{t} \mathbf{Z}\right)=h_{0}(t) \exp \left(\sum_{k=1}^{p} \beta_{k} Z_{k}\right)
$$

O logaritmo de $\frac{h(t \mid \mathbf{Z})}{h_{0}(t)}$ é $\sum_{k=1}^{p} \beta_{k} Z_{k}$ nas formulações tradicionais de modelos lineares com efeitos de covariáveis. Este é o modelo de riscos proporcionais porque, se dois indivíduos com covariáveis de valores $\mathbf{Z}$ e $\mathbf{Z}^{\star}$ forem observados, a razão dos riscos é constante. Esse valor constante é chamado de risco relativo para um indivíduo com os fatores de risco $Z^{\star}$. Particularmente, se $Z_{1}$ indicar o efeito do tratamento $\left(Z_{1}=1\right.$, se for grupo de tratamento e $Z_{1}=0$ se for grupo-controle) e todas as outras covariáveis tiverem os mesmos valores, então $\frac{h(t \mid \mathbf{Z})}{h_{0}(t)}=\exp \left(\beta_{1}\right)$ que é o risco de acontecer o evento se o indivíduo tiver recebido o tratamento (grupo de tratamento), sobre o risco de ter acontecido o evento se o indivíduo for do grupo de controle (grupocontrole).

$$
\frac{h(t \mid \mathbf{Z})}{h\left(t \mid \mathbf{Z}^{*}\right)}=\frac{h_{0}(t) \exp \left(\sum_{k=1}^{p} \beta_{k} Z_{k}\right)}{h_{0}(t) \exp \left(\sum_{k=1}^{p} \beta_{k} Z_{k}^{*}\right)}=\exp \left[\sum_{k=1}^{p} \beta_{k}\left(Z_{k}-Z_{k}^{*}\right)\right]
$$

As saídas da regressão produzidas pelo pacote estatístico SAS fornecem o teste de hipótese para $\beta$ (SAS, 2001). A hipótese nula testada é a de que todos os coeficientes das variáveis adicionadas ao modelo sejam zero. O critério do teste é dado pela estatística qui-quadrado, que é calculada pela diferença entre duas vezes a verossimilhança parcial do modelo com e sem as variáveis de controle, e o p-valor é encontrado utilizando-se como graus de liberdade o número de variáveis do modelo (ALISSON, 2003).

A principal variável de interesse, neste caso, é a educação dos pais. Como é consenso na literatura, a educação dos pais, particularmente a da mãe, apresenta uma associação positiva com a probabilidade de estudar e, espera-se, uma relação negativa com a probabilidade de entrar no mercado de trabalho e de ter o primeiro filho.

Testou-se também se a educação do pai está mais relacionada aos eventos associados à participação na força de trabalho em detrimento, ou menor associação, com a escolaridade e ter o primeiro filho. Incluiu-se a variável de situação de residência até os 15 anos $^{4}$ para verificar se o fato de o indivíduo ter residido em áreas urbanas até essa idade diminui o risco de sair da escola e ter o primeiro filho. As demais variáveis de idade (saída da escola, primeiro filho e entrada no mercado de trabalho) foram incluídas para identificar o sentido da associação existente entre elas.

\footnotetext{
${ }^{4}$ Como o interesse do estudo é também verificar se as transições ocorrem nas idades jovens, a situação de domicílio até os 15 anos (urbano, rural e urbano e rural) é mais importante do que a situação de domicílio (urbano e rural) do entrevistado que tinha entre 20 e 49 anos na época da entrevista.
} 
QUADRO 1

Variáveis explicativas inclusas nos modelos: entrada no mercado de trabalho, saída da escola e primeiro filho

\begin{tabular}{|c|c|c|c|c|c|}
\hline Variáveis & Descrição & Valores & $\begin{array}{c}\text { Entrar no } \\
\text { mercado de } \\
\text { trabalho }\end{array}$ & $\begin{array}{l}\text { Sair da } \\
\text { escola }\end{array}$ & $\begin{array}{l}\text { Primeiro } \\
\text { filho }\end{array}$ \\
\hline edpai & Nível de educação do pai & 1 - Nenhuma educação & $x$ & $x$ & $x$ \\
\hline edmae & Nível de educação da mãe & $\begin{array}{l}2 \text { - Ensino fundamental } \\
3 \text { - Ensino Médio } \\
4 \text { - Superior e mais }\end{array}$ & $\mathrm{x}$ & $\mathrm{x}$ & $\mathrm{X}$ \\
\hline urb15 & Área em que viveu até 15 anos & $\begin{array}{l}1 \text { - Só urbano } \\
0 \text { - Demais áreas }\end{array}$ & $\mathrm{x}$ & $x$ & $x$ \\
\hline idadesc & Idade de saída da escola & Contínua & $\mathrm{x}$ & & $x$ \\
\hline idadfil1 & Idade ao ter o primeiro filho & Contínua & & $\mathrm{x}$ & \\
\hline idtrab & Idade de entrada no mercado de trabalho & Contínua & & $\mathrm{x}$ & $\mathrm{X}$ \\
\hline
\end{tabular}

Fonte: PPV - IBGE/Banco Mundial.

TABELA 1

Distribuição da amostra, por sexo, segundo idade Regiões Sudeste e Nordeste do Brasil - 1996-1997

\begin{tabular}{cccc}
\hline Idade & Homens & Mulheres & Total \\
\hline 20-24 anos & 747 & 832 & 1.579 \\
25-29 anos & 660 & 756 & 1.416 \\
30-34 anos & 642 & 697 & 1.339 \\
35-39 anos & 550 & 597 & 1.147 \\
40-44 anos & 455 & 559 & 1.014 \\
45-49 anos & 391 & 403 & 794 \\
Total & $\mathbf{3 . 4 4 5}$ & $\mathbf{3 . 8 4 4}$ & $\mathbf{7 . 2 8 9}$ \\
\hline
\end{tabular}

Fonte: Elaborado pela autora, a partir dos microdados da PPV - IBGE/Banco Mundial.

\section{Descrição da amostra}

Para efeito de padronização da amostra, foram eliminados do banco de dados valores inconsistentes e todos os indivíduos que tinham menos de 20 e mais de 49 anos na época da entrevista. A distribuição da amostra por sexo e idade consta na Tabela 1.

Relação entre a idade de entrada no mercado de trabalho e a idade de saída da escola

Esta seção teve como propósito verificar se existe alguma associação entre a idade de entrada no mercado de trabalho e a idade de saída da escola. A Tabela 2 indica que, para os homens de até 19 anos, 69,84\% já saíram da escola e $90,97 \%$ já entraram no mercado de trabalho. Uma análise mais acurada da tabela de contingência indica que a entrada no mercado de trabalho é um evento que precede a saída da escola e pode acionar essa transição, pois, dos $68,88 \%$ que entraram no mercado de trabalho até os 15 anos, 36,95\% saíram da escola na mesma idade, $16,55 \%$ com 16 a 19 anos e $11,79 \%$ após os 19 anos. Além disso, as proporções a partir e acima da diagonal principal são significativamente maiores em relação às células abaixo da diagonal principal. A própria configuração da tabela e a estatística qui-quadrado estimada revelam que não se trata de processos independentes.

Ressalta-se que a transição para o mercado de trabalho é muito intensa até a idade de 15 anos, se for considerado que a idade legal, no Brasil, para inserção na atividade econômica é 16 anos. 
TABELA 2

Distribuição dos homens, por idade de saída da escola, segundo idade de entrada no mercado de trabalho Regiões Sudeste e Nordeste do Brasil - 1996-1997

Em porcentagem

\begin{tabular}{|c|c|c|c|c|c|c|}
\hline \multirow{2}{*}{$\begin{array}{l}\text { Entrada no mercado } \\
\text { de trabalho }\end{array}$} & \multicolumn{6}{|c|}{ Saída da escola } \\
\hline & $\begin{array}{l}\text { Não } \\
\text { saíram }\end{array}$ & $\begin{array}{l}\text { Até } 15 \\
\text { anos }\end{array}$ & $\begin{array}{l}\text { De } 16 \text { a } \\
19 \text { anos }\end{array}$ & $\begin{array}{l}\text { De } 20 \text { a } \\
24 \text { anos }\end{array}$ & $\begin{array}{l}\text { Mais de } \\
25 \text { anos }\end{array}$ & Total \\
\hline Não entraram & 1,04 & 0,26 & 0,26 & 0.35 & 0.00 & 1.92 \\
\hline Até 15 anos & 3,60 & 36,95 & 16,55 & 8,16 & 3,63 & 68,88 \\
\hline 16 a 19 anos & 2,29 & 5,66 & 7,40 & 4,91 & 1,83 & 22,09 \\
\hline 20 a 24 anos & 1,19 & 0,99 & 1,63 & 1,86 & 0,64 & 6,30 \\
\hline Mais de 25 anos & 0,12 & 0,12 & 0,03 & 0,29 & 0,26 & 0,81 \\
\hline Total & 8,24 & 43,98 & 25,86 & $15,56 \%$ & 6,36 & 100,00 \\
\hline
\end{tabular}

Fonte: Elaborado pela autora, a partir dos microdados da PPV - IBGE/Banco Mundial. Nota: Teste do qui-quadrado significativo com probabilidade $<0.0001$ (=554.97 - $16 \mathrm{gl}$ ).

TABELA 3

Distribuição das mulheres, por idade de saída da escola, segundo a idade de entrada no mercado de trabalho

Em porcentagem

\begin{tabular}{lrrrrrr}
\hline & \multicolumn{7}{c}{ Saída da escola } \\
\cline { 2 - 7 } $\begin{array}{l}\text { Entrada no mercado } \\
\text { de trabalho }\end{array}$ & $\begin{array}{c}\text { Não } \\
\text { saíram }\end{array}$ & $\begin{array}{c}\text { Até 15 } \\
\text { anos }\end{array}$ & $\begin{array}{c}\text { De 16 a } \\
\mathbf{1 9} \text { anos }\end{array}$ & $\begin{array}{c}\text { De 20 a } \\
\mathbf{2 4} \text { anos }\end{array}$ & $\begin{array}{c}\text { Mais de } \\
\mathbf{2 5} \text { anos }\end{array}$ & Total \\
\hline Não entraram & $\mathbf{1 , 9 5}$ & 5,62 & 2,37 & $\mathbf{1 , 1 4}$ & 0,16 & 11,24 \\
Até 15 anos & 2,71 & 25,39 & 10,30 & 5,62 & 2,16 & 46,18 \\
16 a 19 anos & 2,68 & 5,98 & 8,64 & 6,09 & 1,95 & 25,34 \\
20 a 24 anos & $\mathbf{1 , 2 7}$ & 2,19 & 3,69 & 3,75 & 1,30 & 12,20 \\
Mais de 25 anos & 0,26 & 1,93 & 1,01 & 1,09 & 0,75 & 5,05 \\
Total & $\mathbf{8 , 8 7}$ & $\mathbf{4 1 , 1 0}$ & $\mathbf{2 6 , 0 1}$ & $\mathbf{1 7 , 6 9}$ & $\mathbf{6 , 3 2}$ & $\mathbf{1 0 0 , 0 0}$ \\
\hline
\end{tabular}

Fonte: Elaborado pela autora, a partir dos microdados da PPV - IBGE/Banco Mundial.

Nota: Teste do qui-quadrado significativo com probabilidade $<0.0001$ (=507.33 - $16 \mathrm{gl})$.

A Tabela 3 revela que a mulher permanece mais tempo fora do mercado de trabaIho e uma proporção delas jamais se insere na atividade econômica, embora o padrão de saída da escola seja bastante similar ao dos homens. Como no caso masculino, os dados sugerem que, também para as muIheres, a entrada no mercado de trabalho precede a saída da escola e pode acionar esse processo. A estatística qui-quadrado estimada também revela que os processos não são independentes para as mulheres.

Relação entre a idade de entrada no mercado de trabalho e a idade ao primeiro filho

Este tópico e o próximo referem-se apenas às mulheres e analisam a relação entre a idade de entrada no mercado de trabalho e a ocorrência do primeiro filho, e entre a idade de saída da escola e a ocorrência do primeiro filho, nessa ordem. Evidencia-se, pela Tabela 4, que entrar no mercado de trabalho é uma decisão que precede a de ter filhos. Note-se que, das mulheres que entraram no mercado de trabalho até os 15 anos (46,18\%), uma maior proporção delas (15,14\%) tiveram filhos apenas aos $20-24$ anos, $11,86 \%$ dos 16 aos 19 anos e somente $1,22 \%$ aos 15 anos. Esses resultados indicam uma tendência similar ao comportamento europeu, ou seja, primeiro se busca a independência financeira para, posteriormente, assumir os papéis relacionados à fecundidade, o que tem aumentado a idade ao ter o primeiro filho. Pela própria seqüência de acontecimento desses eventos, é notório que existe uma associação entre eles, o que é corroborado pela estatística qui-quadrado. 
TABELA4

Distribuição das mulheres, por idade ao ter o primeiro filho, segundo a idade de entrada no mercado de trabalho Regiões Sudeste e Nordeste do Brasil - 1996-1997

Em porcentagem

\begin{tabular}{lcccccc}
\hline \multirow{2}{*}{$\begin{array}{l}\text { Entrada no mercado } \\
\text { de trabalho }\end{array}$} & $\begin{array}{c}\text { Saída da escola } \\
\text { saíram }\end{array}$ & $\begin{array}{c}\text { Até 15 } \\
\text { anos }\end{array}$ & $\begin{array}{c}\text { De 16 a } \\
\mathbf{1 9} \text { anos }\end{array}$ & $\begin{array}{c}\text { De 20 a } \\
\mathbf{2 4} \text { anos }\end{array}$ & $\begin{array}{c}\text { Mais de } \\
\mathbf{2 5} \text { anos }\end{array}$ & Total \\
\cline { 2 - 7 } & 3,56 & 0,49 & 3,04 & 3,02 & 1,12 & 11,24 \\
Não entraram & 9,52 & 1,22 & 11,86 & 15,14 & 8,43 & 46,18 \\
Até 15 anos & 8,61 & 0,29 & 3,59 & 7,18 & 5,67 & 25,34 \\
16 a 19 anos & 5,75 & 0,21 & 1,30 & 2,45 & 2,50 & 12,20 \\
20 a 24 anos & 1,12 & 0,10 & 1,20 & 1,59 & 1,04 & 5,05 \\
Mais de 25 anos & $\mathbf{2 8 , 5 6}$ & $\mathbf{2 , 3 2}$ & $\mathbf{2 0 , 9 9}$ & $\mathbf{2 9 , 3 7}$ & $\mathbf{1 8 , 7 6}$ & $\mathbf{1 0 0 , 0 0}$ \\
\hline Total & &
\end{tabular}

Fonte: Elaborado pela autora, a partir dos microdados da PPV - IBGE/Banco Mundial.

Nota: Teste do qui-quadrado significativo com probabilidade $<0.0001$ (=246.68 - $16 \mathrm{gl}$ ).

TABELA 5

Distribuição das mulheres, por idade ao ter o primeiro filho, segundo a idade de saída da escola Regiões Sudeste e Nordeste do Brasil - 1996-1997

Em porcentagem

\begin{tabular}{lcccccr}
\hline \multirow{2}{*}{$\begin{array}{l}\text { Entrada no mercado } \\
\text { de trabalho }\end{array}$} & $\begin{array}{c}\text { Não } \\
\text { saíram }\end{array}$ & $\begin{array}{c}\text { Até 15 } \\
\text { anos }\end{array}$ & $\begin{array}{c}\text { De 16 a } \\
\mathbf{1 9} \text { anos }\end{array}$ & $\begin{array}{r}\text { De 20 a } \\
\mathbf{2 4} \text { anos }\end{array}$ & $\begin{array}{c}\text { Mais de } \\
\mathbf{2 5} \text { anos }\end{array}$ & Total \\
\cline { 2 - 7 } & 6,53 & 0,18 & 0,83 & 0,81 & 0,52 & 8,87 \\
Não saíram & 6,04 & 1,77 & 13,66 & 13,66 & 5,98 & 41,10 \\
Até 15 anos & 7,28 & 0,16 & 4,55 & 8,69 & 5,33 & 26,01 \\
16 a 19 anos & 6,79 & 0,21 & 1,20 & 4,73 & 4,76 & 17,69 \\
20 a 24 anos & 1,93 & 0,00 & 0,75 & 1,48 & 2,16 & 6,32 \\
Mais de 25 anos & $\mathbf{2 8 , 5 6}$ & $\mathbf{2 , 3 2}$ & $\mathbf{2 0 , 9 9}$ & $\mathbf{2 9 , 3 7}$ & $\mathbf{1 8 , 7 6}$ & $\mathbf{1 0 0 , 0 0}$ \\
Total & &
\end{tabular}

Fonte: Elaborado pela autora, a partir dos microdados da PPV - IBGE/Banco Mundial.

Nota: Teste do qui-quadrado significativo com probabilidade $<0.0001$ (=804.08 - $16 \mathrm{gl})$.

Relação entre a idade de saída da escola e a idade ao ter o primeiro filho

Os resultados apresentados na Tabela 5 indicam que as jovens de até 19 anos saem da escola para depois terem o primeiro filho. Os dados parecem mostrar que a transição para o primeiro filho, como um processo que aciona a transição para fora da escola, é importante apenas para quem teve esse filho até os 15 anos, do que se pode inferir, grosso modo, tratar-se de um evento não planejado. Das 2,32\% jovens que tiveram filhos até os 15 anos, $1,77 \%$ deixaram a escola nessa idade. Da mesma forma que a decisão de entrar no mercado de trabalho precede a de ter o primeiro filho, a saída da escola também acontece antes desse evento. Entretanto, deve-se ressaltar que, quanto mais cedo a jovem sai da escola, mais nova ela terá o primeiro filho, pois, entre as que saíram da escola até os 15 anos, $13,66 \%$ tiveram o primeiro filho entre 16 e 19 anos e a mesma proporção o tiveram entre 20 e 24 anos. Tendo em vista que para as mulheres a entrada no mercado de trabalho, em maior proporção, precede a saída da escola ou aciona esta saída, uma seqüência desses eventos seria: a jovem estudante decide entrar no mercado de trabalho e, por isso, deixa a escola, decidindo, algum tempo depois, ter filhos. É notório que todos esses processos estão fortemente associados, contudo, no escopo deste trabalho, nada mais se pode dizer sobre tal associação.

\section{Funções de sobrevivência e risco}

Nesta seção, foram geradas tábuas de vida por sexo, elaborando-se, a partir delas, gráficos para uma melhor análise da função 
de sobrevivência em cada um dos eventos estudados.

Foram destacadas nos gráficos medidas de posição, como a mediana, o primeiro e o terceiro quartis, conforme a fórmula seguinte:

Mediana:

$$
x_{0,5}=i d_{\text {inf }}-\left(\frac{\left(S\left(i d_{\text {inf }}\right)-0,5\right) *\left(i d_{\text {inf }}-i d_{\text {sup }}\right)}{S\left(i d_{\text {inf }}\right)-S\left(i d_{\text {sup }}\right)}\right)
$$

Idade de entrada no mercado de trabalho

A análise das curvas de sobrevivência por sexo e a função do risco de entrar no mercado de trabalho demonstram que essa transição ocorre, tanto para os homens quanto para as mulheres, em idade jovem

\section{GRÁFICO 1}

Curvas de sobrevivência na inatividade, segundo sexo

Regiões Sudeste e Nordeste do Brasil - 1996-1997

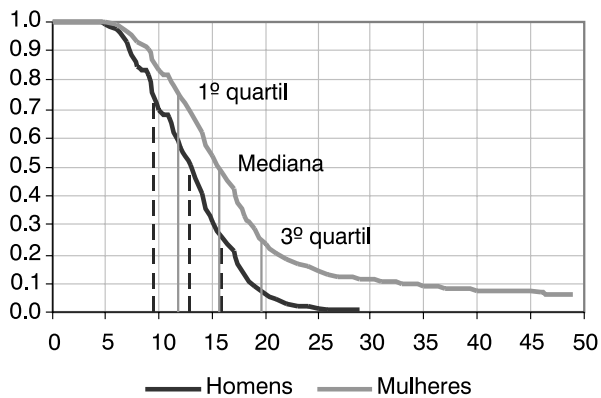

Fonte: Elaborado pela autora, a partir dos microdados da PPV - IBGE/Banco Mundial.

GRÁFICO 3

Curvas de sobrevivência na escola, segundo sexo Regiões Sudeste e Nordeste do Brasil - 1996-1997

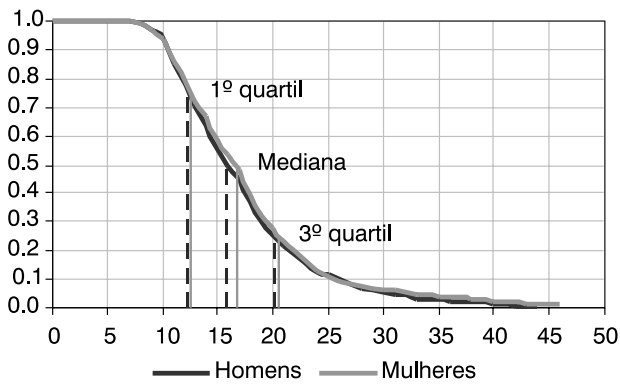

Fonte: Elaborado pela autora, a partir dos microdados da PPV - IBGE/Banco Mundial.
(Gráficos 1 e 2). Note-se que os maiores riscos de entrada no mercado de trabalho, para ambos os sexos, apresentam tendência de crescimento até próximo da idade pertencente ao terceiro quartil de transição. Registra-se, também, como já verificado anteriormente, uma defasagem na idade mediana de inserção: 15,66 anos para as mulheres e 13,08 anos para os homens. Com 19,65 anos, $75 \%$ das mulheres já se inseriram no mercado de trabalho, enquanto para os homens essa idade corresponde a 16,10 anos.

Idade de saída da escola

A idade de saída da escola, conforme os Gráficos 3 e 4, não apresenta variabi-

GRÁFICO 2

Função do risco de entrar no mercado de trabalho, segundo sexo

Regiões Sudeste e Nordeste do Brasil - 1996-1997

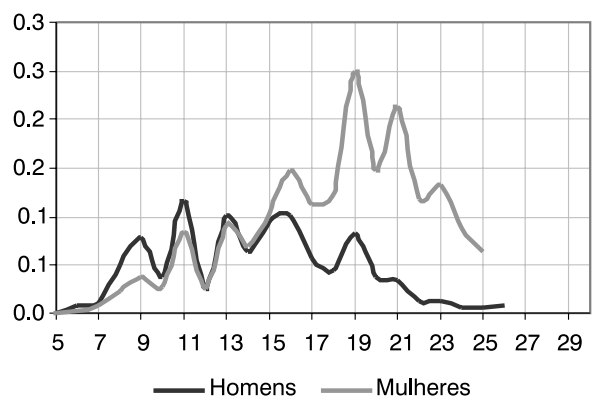

Fonte: Elaborado pela autora, a partir dos microdade da PPV - IBGE/Banco Mundial.

GRÁFICO 4

Função do risco de sair da escola, segundo sexo Regiões Sudeste e Nordeste do Brasil - 1996-1997

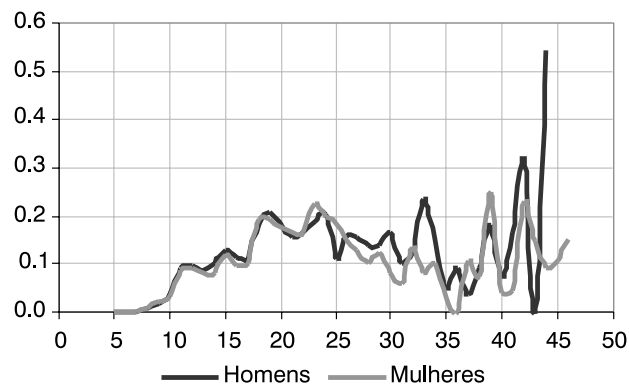

Fonte: Elaborado pela autora, a partir dos microdados da PPV - IBGE/Banco Mundial. 
lidade entre homens e mulheres, conseqüentemente, o padrão de risco é também similar para ambos. ${ }^{5}$ Destaca-se que a transição ocorre majoritariamente em idade jovem: para os homens, a idade mediana é de 15,97 anos e, para as mulheres, 16,69 anos. Com 20 anos, $75 \%$ de homens e mulheres já saíram da escola, e o risco de saída é crescente até próximo dos 20 anos.

\section{Nascimento do primeiro filho}

A decisão de ter o primeiro filho é posterior à entrada no mercado de trabalho e à saída da escola. De acordo com a

\section{GRÁFICO 5}

Curvas de sobrevivência para as mulheres, segundo a idade ao ter o primeiro filho

Regióes Sudeste e Nordeste do Brasil - 1996-1997

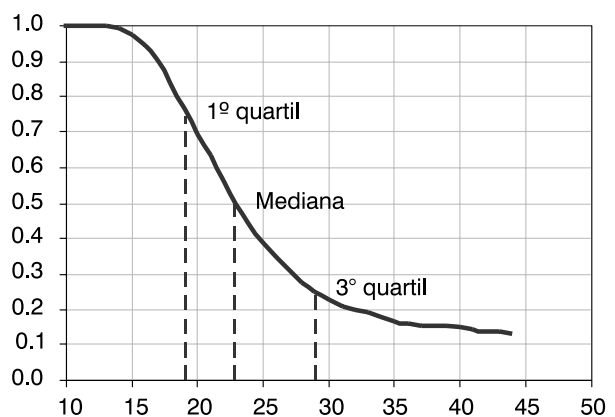

Fonte: Elaborado pela autora, a partir dos microdados da PPV - IBGE/Banco Mundial.

GRÁFICO 7

Curvas de sobrevivência para os homens, segundo idade de entrada no mercado de trabalho e saída da escola

Regiões Sudeste e Nordeste do Brasil - 1996-1997

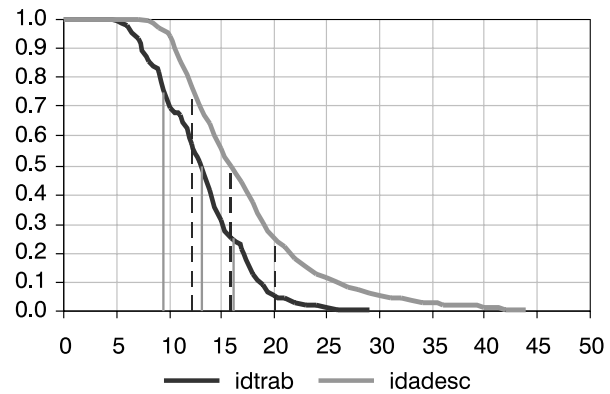

Fonte: Elaborado pela autora, a partir dos microdados da PPV - IBGE/Banco Mundial. função de sobrevivência, mostrada no Gráfico 5, a idade no primeiro quartil é 19,24 anos e a idade mediana corresponde a 23 anos, contra 11,86 e 15,66 anos da entrada no mercado de trabalho e 12,57 e 16,69 anos de saída da escola, relativas ao primeiro quartil e mediana, respectivamente. O risco de se ter o primeiro filho é crescente até próximo dos 25 anos, passando a diminuir a partir dessa idade (Gráfico 6).

O Gráfico 7 apresenta as curvas de sobrevivência na escola e na inatividade para os homens, enquanto o Gráfico 8 traz as curvas de sobrevivência na escola, inatividade e ter o primeiro filho para as

\section{GRÁFICO 6}

Função do risco para as mulheres de ter o primeiro filho

Regióes Sudeste e Nordeste do Brasil - 1996-1997

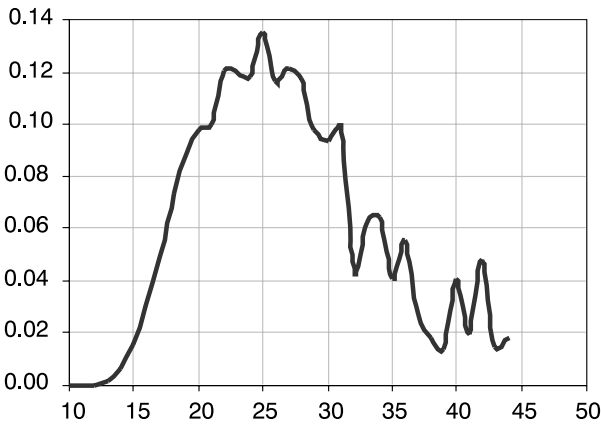

Fonte: Elaborado pela autora, a partir dos microdados da PPV - IBGE/Banco Mundial.

\section{GRÁFICO 8}

Curvas de sobrevivência para as mulheres, segundo idade de entrada no mercado de trabalho, saída da escola e ao ter o primeiro filho

Regiões Sudeste e Nordeste do Brasil - 1996-1997

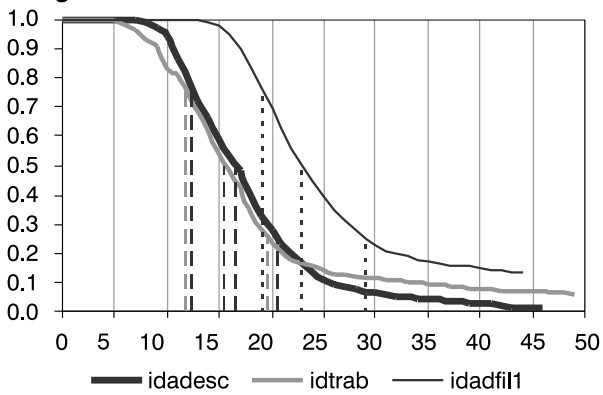

Fonte: Elaborado pela autora, a partir dos microdados da PPV - IBGE/Banco Mundial.

\footnotetext{
${ }^{5}$ É provável que o padrão de escolaridade que apresenta vantagens educacionais para as mulheres seja coerente com as coortes mais jovens relativamente àquelas pesquisadas pela PPV.
} 
mulheres. Nota-se que a entrada no mercado de trabalho é transição que antecede a saída da escola para ambos os sexos. No caso dos homens, parece haver uma certa simultaneidade entre escola e trabalho, ou seja, a transição para o mercado de trabalho não aciona, de imediato, a transição para fora da escola. Já no caso das mulheres, parece que a entrada no mercado de trabaIho provoca, quase que imediatamente, a saída da escola. No entanto, a decisão de ter o primeiro filho sucede a saída da escola com uma defasagem relevante.

\section{Análise do efeito das variáveis selecionadas sobre o risco}

As regressões que medem o risco proporcional segundo covariáveis selecionadas foram operacionalizadas para homens e mulheres separadamente. $\mathrm{O}$ propósito da desagregação é verificar se alguma variável apresenta um fator de risco maior ou menor relacionado ao sexo do indivíduo exposto ao risco. ${ }^{6}$

Nota-se, na Tabela 6, que a educação do pai tem um efeito significativo na redução do risco de os homens entrarem no mercado de trabalho. Como se vê, a cada nível educacional mais alto, em contraposição a nenhuma educação, o risco é menor. A variável educação da mãe, por sua vez, apesar de apresentar efeito menor, relativamente à educação do pai, na redução do risco de o homem entrar no mercado de trabalho, possui impacto significativo para as mulheres, embora em magnitude menor do que o verificado para os homens. A educação do pai não apresentou efeito importante sobre o risco de a mulher entrar no mercado de trabalho.

O fato de o indivíduo ter vivido em área urbana até a idade de 15 anos tem impacto significativo no risco relacionado à entrada no mercado de trabalho. Note-se que para o indivíduo do sexo masculino que viveu somente em área urbana até os 15 anos, o risco corresponde a apenas 55,9\% daquele verificado para os que viveram em outras áreas. Esse impacto é menor quando se trata de mulheres, sendo que o risco daquelas com domicílio urbano é $89,1 \%$ relativamente ao das que residiram em outras áreas. A idade de saída da escola (idadesc) tem efeito significativo e reduz em $2,5 \%$ e $0,4 \%$ o risco de homens e mulheres, respectivamente, entrarem no mercado de trabalho. Cada ano adicional na escola, controlando-se pelas demais variáveis, produz um efeito positivo para ambos os sexos. Quanto mais se prolonga a permanência na escola, em detrimento da entrada no mercado de trabalho, pode-se supor que o estoque de capital humano acumulado por meio da educação formal garantirá melhor inserção no mercado de trabalho.

TABELA 6

Efeito das variáveis selecionadas sobre o risco de entrar no mercado de trabalho Regiões Sudeste e Nordeste do Brasil - 1996-1997

\begin{tabular}{lcccc}
\hline & \multicolumn{4}{c}{ Entrada no mercado de trabalho } \\
\cline { 2 - 5 } Variáveis & P-valor & Homem & P-valor & Mulher \\
\hline edpai2 & 0.0015 & 87.3 & 0.7722 & 98.8 \\
edpai3 & $<.0001$ & 68.6 & 0.2694 & 93.0 \\
edpai4 & $<.0001$ & 57.5 & 0.3534 & 90.6 \\
edmae2 & 0.0120 & 89.9 & 0.0125 & 90.1 \\
edmae3 & $<.0001$ & 73.5 & 0.0004 & 79.5 \\
edmae4 & 0.0006 & 62.2 & 0.0600 & 75.4 \\
urb15 & $<.0001$ & 55.9 & 0.0033 & 89.1 \\
idadesc & $<.0001$ & -2.5 & 0.2206 & -0.4 \\
Idadfil1 & - & - & 0.0081 & -0.1 \\
\hline
\end{tabular}

Fonte: Elaborado pela autora, a partir dos microdados da PPV - IBGE/Banco Mundial. Nota: Foram omitidas as variáveis edpai1 e edmae1.

${ }^{6}$ A Tabela 9 apresenta todos os parâmetros calculados pelo modelo. 
No caso das mulheres, a idade ao ter primeiro filho reduz em apenas $0,1 \%$ o risco de entrar no mercado de trabalho.

A educação da mãe é altamente significativa na redução do risco de sair da escola para homens e mulheres. Registrase, conforme a Tabela 7, que filhos de mães com educação fundamental apresentam $84,1 \%$ do risco de sair da escola daqueles cujas mães não acumularam nenhum grau educacional. Cada nível educacional da mãe, em contraste com a categoria omitida (nenhuma educação), reduz o risco de os homens e as mulheres saírem da escola, embora o efeito sobre a diminuição do risco de as mulheres saírem da escola ser, em magnitude, maior do que o verificado para os homens. A educação do pai também foi significante na diminuição do risco de homens e mulheres saírem da escola, embora o efeito se apresente maior para os homens. Parece haver algum viés de sexo na associação da educação dos pais sobre o risco de sair da escola, ou seja, o efeito da educação da mãe é maior para as mulheres e o da educação do pai é maior para os homens.

O risco de sair da escola dos indivíduos do sexo masculino que residiram exclusivamente em áreas urbanas até a idade de 15 anos é de $88 \%$ daquele verificado para os que residiram em outras áreas. No caso das mulheres, o risco corresponde a $76,6 \%$ do observado para as que viveram em outras áreas.
Cada ano a mais que se posterga a entrada no mercado de trabalho reduz em $2,3 \%$ o risco de sair da escola, para os homens, e em $0,3 \%$, para as mulheres. A idade ao ter o primeiro filho diminui o risco de as mulheres saírem da escola em $0,8 \%$, ou seja, cada ano adicional da idade ao ter o primeiro filho reduz o risco das mulheres saírem da escola. Embora o porcentual de redução seja relativamente baixo, este resultado é coerente com Stupp e Cáceres (2001), cujo trabalho demonstrou que a idade com a qual a mulher deixa a escola, e não o mais alto nível educacional, é o fator crítico para a transição para o primeiro filho.

A educação da mãe apresenta efeito positivo na redução do risco de ter o primeiro filho (Tabela 8). Enquanto filhas de mães com educação fundamental apresentam $90,5 \%$ do risco de ter o primeiro filho daquelas cujas mães não possuem nenhuma educação, filhas de mães com nível superior ou mais registram risco $32,1 \%$ menor do que o das filhas cujos pais não têm nenhum nível educacional. A educação do pai só apresenta efeito positivo na redução do risco de ter o primeiro filho para os níveis educacionais fundamental e médio.

A idade de entrada no mercado de trabalho aumenta em $3,8 \%$ o risco de ter o primeiro filho, enquanto a idade de saída da escola reduz em 2,2\% esse risco. Tais resultados são bastante plausíveis e indicam que, quanto mais tardiamente se entra no mercado de trabalho, por hipótese,

TABELA 7

Efeito das variáveis selecionadas sobre o risco de sair da escola Regiões Sudeste e Nordeste do Brasil - 1996-1997

\begin{tabular}{lcccc}
\hline & \multicolumn{4}{c}{ Saída da escola } \\
\cline { 2 - 5 } Variáveis & P-valor & Homem & P-valor & Mulher \\
\hline edpai2 & 0.0004 & 85.9 & 0.0553 & 92.4 \\
edpai3 & $<.0001$ & 71,0 & 0.0001 & 77.6 \\
edpai4 & $<.0001$ & 52.1 & $<.0001$ & 64.3 \\
edmae2 & $<.0001$ & 84.1 & $<.0001$ & 80.4 \\
edmae3 & $<.0001$ & 65.3 & $<.0001$ & 63,0 \\
edmae4 & $<.0001$ & 53.5 & $<.0001$ & 49.6 \\
urb15 & $<.0001$ & 76.6 & $<.0001$ & 79.1 \\
idtrab & $<.0001$ & -2.3 & 0.1782 & -0.3 \\
Idadfil1 & - & 0 & $<.0001$ & -0.8 \\
\hline
\end{tabular}

Fonte: Elaborado pela autora, a partir dos microdados da PPV - IBGE/Banco Mundial. Nota: Foram omitidas as variáveis edpai1 e edmae1. 
TABELA 8

Efeito das variáveis selecionadas sobre o risco de ter o primeiro filho Regiões Sudeste e Nordeste do Brasil - 1996-1997

\begin{tabular}{lcc}
\hline Variáveis & p-valor & Razão de Risco( \%) \\
\hline edpai2 & 0.0447 & 92.3 \\
edpai3 & 0.0056 & 84.1 \\
edpai4 & 0.2050 & 88.1 \\
edmae2 & 0.0117 & 90.5 \\
edmae3 & 0.0005 & 80.8 \\
edmae4 & 0.0058 & 67.9 \\
urb15 & 0.4959 & 97.5 \\
idtrab & $<.0001$ & 3.8 \\
Idadesc & $<.0001$ & -2.2 \\
\hline
\end{tabular}

Fonte: Elaborado pela autora, a partir dos microdados da PPV - IBGE/Banco Mundial.

Nota: Foram omitidas as variáveis edpai1 e edmae1.

TABELA 9

Saídas de regressão

\begin{tabular}{|c|c|c|c|c|c|c|}
\hline & \multicolumn{6}{|c|}{ Entrada no mercado de trabalho } \\
\hline & \multicolumn{3}{|c|}{ Homem } & \multicolumn{3}{|c|}{ Mulher } \\
\hline Total de eventos & \multicolumn{3}{|c|}{597} & \multicolumn{3}{|c|}{721} \\
\hline censura & \multirow{2}{*}{\multicolumn{3}{|c|}{21}} & \multicolumn{3}{|c|}{64} \\
\hline Ajuste do modelo & & & & & & \\
\hline$(-) 2 \log L c / \operatorname{cov}$ & & \multicolumn{2}{|c|}{7650.28} & \\
\hline (-) $2 \log \mathrm{L} s / \mathrm{cov}$ & \multicolumn{2}{|r|}{6291.23} & & \multicolumn{3}{|c|}{7608.16} \\
\hline Razão de Verosimilhança = Qui-quadrado & \multicolumn{3}{|c|}{123.46} & \multicolumn{3}{|c|}{42.12} \\
\hline $\operatorname{Pr}>$ qui-quadrado & \multicolumn{3}{|c|}{$<.0001$} & \multicolumn{3}{|c|}{$<.0001$} \\
\hline Variáveis & $\begin{array}{l}\text { Parâmetro } \\
\text { estimado }\end{array}$ & $\begin{array}{c}\text { Pr }>\text { Qui- } \\
\text { qua }\end{array}$ & $\begin{array}{c}\text { Razão } \\
\text { de risco }\end{array}$ & $\begin{array}{l}\text { Parâmetro } \\
\text { estimado }\end{array}$ & $\begin{array}{c}\text { Pr>Qui- } \\
\text { qua }\end{array}$ & $\begin{array}{l}\text { Razão } \\
\text { de risco }\end{array}$ \\
\hline edpai & -0.1372 & 0.0648 & 0.8720 & -0.0168 & 0.7966 & 0.9830 \\
\hline \multirow{2}{*}{ edmae } & $\begin{array}{r}0.0743 \\
-0.1432\end{array}$ & 0.0954 & 0.8670 & $\begin{array}{r}0.0650 \\
-0.1388\end{array}$ & 0.0848 & 0.8700 \\
\hline & 0.0859 & 0.0004 & 0.0070 & 0.0805 & 0.0040 & 0.0700 \\
\hline \multirow[t]{2}{*}{ urb15 } & -0.4575 & 0.0001 & 0.6330 & -0.2327 & 0.0169 & 0.7920 \\
\hline & 0.1184 & & & 0.0974 & & \\
\hline \multirow[t]{2}{*}{ mun } & -0.1022 & 0.2376 & 0.9030 & -0.1002 & 0.2248 & 0.9050 \\
\hline & 0.0865 & & & 0.0825 & & \\
\hline \multirow[t]{2}{*}{ catocuppai } & -0.1012 & 0.1337 & 0.9040 & -0.0064 & 0.9196 & 0.9940 \\
\hline & 0.0675 & & & 0.0634 & & \\
\hline \multirow[t]{2}{*}{ catocupmae } & -0.1103 & 0.1934 & 0.8960 & -0.0892 & 0.2659 & 0.9150 \\
\hline & 0.0848 & & & 0.0802 & & \\
\hline \multirow[t]{2}{*}{ Idadesc } & -0.0396 & 0.0001 & 0.9610 & -0.0180 & 0.0417 & 0.9820 \\
\hline & 0.0095 & & & 0.0088 & & \\
\hline
\end{tabular}

Fonte: Elaborado pela autora, a partir dos dados da PPV - IBGE/ Banco Mundial.

mais o indivíduo já teria completado um bom nível educacional e estaria transitando para uma etapa de autonomia financeira coerente com os compromissos relativos à formação de família. Por outro lado, quanto mais anos ele se mantém na escola, ou seja, quanto mais anos se assume os papéis sociais do estudante, mais se atrasa a transição para se assumir os papéis e as despesas relativas à formação de família.

\section{Conclusão}

Mesmo sem nenhuma inferência de causalidade, pode-se esperar que, no Brasil, a mulher primeiro decide trabalhar, quase simultaneamente sai da escola e somente algum tempo depois decide ter o primeiro filho. No caso dos homens, a idade de entrada no mercado de trabalho precede a idade de saída da escola e sugere uma 
possível simultaneidade entre a escola e o trabalho.

Pode-se afirmar que, no Brasil, essas transições são inerentes aos jovens. Em que pese o fato de a transição para ter o primeiro filho suceder as demais transições femininas, baseando-se nesses dados, não parece haver uma tendência clara de aumento da idade dessa transição como citado na literatura européia.

A análise semiparamétrica, entretanto, esclarece alguns pontos com relação a fatores de risco relevantes para cada evento estudado. A educação da mãe mostrou-se fator importante na redução do risco de todos os eventos estudados, corroborando vários estudos da literatura tanto nacional quanto internacional, que encontram evidências de uma associação positiva entre educação dos pais e a opção pela escola. Verificou-se, ainda, maior significância da educação do pai na redução do risco de os homens entrarem no mercado de trabalho, enquanto a educação da mãe está mais relacionada à redução do risco de sair da escola (CORSEIL, SANTOS e FOGUEL, 2001; LEME E WAJNMAN, 2000, KASSOUF, 2001).

Quanto mais se permanece na escola, menor o risco de se entrar no mercado de trabalho e, no caso das mulheres, de ter o primeiro filho. Embora a redução porcentual do risco seja, em todos os casos, abaixo de $5 \%$, essas relações são corroboradas por estudos que demonstram evidência, para a Europa, do aumento do tempo gasto na educação e da entrada no processo de formação de família (COPPOLA e AASSVE, 2003). Isso também é mostrado por estudos como o de Billari et al. (2000) que encontra incompatibilidade do papel de estudante com a transição para a formação de família, o que pressupõe primeiro a saída da escola, tendo o mercado de trabalho como transição intermediária para o processo de formação de família, situação que requer determinada estrutura de gastos incompatíveis com o ciclo de vida estudantil.

É importante ressaltar que tais resultados refletem a experiência de transição das pessoas que tinham idade entre $20 \mathrm{e}$ 49 anos na época da pesquisa (1996-1997), portanto, não necessariamente traduzem a experiência dos jovens que estão realizando as transições nesta década. A importância dos achados deste estudo está justamente nessa característica, pois, se para a experiência dessas coortes fica evidente uma seqüência de transições e que a idade ao ter o primeiro filho reduz em apenas $0,8 \%$ o risco de deixar a escola, é porque a transição para o primeiro filho não representava o principal fator de risco de deixar a escola. Nesse contexto, deve-se avaliar quais são os desafios enfrentados pelos jovens em idade de transição nesta primeira década do século XXI e, a partir de evidências de coortes anteriores, desenhar as políticas apropriadas.

Segundo Camarano et al. (2003), em 2001, a idade média de formação de família era de 22,28 anos para homens e 21,40 anos para mulheres. Em que pese este trabalho não trazer informações sobre a idade masculina de formação de família, contudo, a idade média das mulheres que compõem a amostra é 22,09 anos, ou seja, praticamente não mudou, feitas as devidas ressalvas sobre a abrangência das duas fontes (PNAD e PPV). Por outro lado, a idade média de entrada na População Economicamente Ativa-PEA, em 2001, foi de 20,2 anos para os homens e 20,16 anos para as mulheres, enquanto a idade média de entrada verificada para os indivíduos que compõem a amostra deste trabalho foi de 13,4 e 15,71 anos, respectivamente, para os sexos masculino e feminino. ${ }^{7}$ Em que pese a amostra ser representativa apenas para as Regiões Nordeste e Sudeste, parte do aumento de idade de entrada na PEA verificado pode ser devido às mudanças no perfil da demanda por mão-de-obra, resultante da reestruturação produtiva iniciada na década de 90 , que tornou o mercado de trabalho mais seletivo. Isso pode ter dificultado a obtenção do primeiro emprego (CAMARANO et al., 2003).

Uma vez identificados os desafios, as idades e os fatores intervenientes nas

${ }^{7}$ Tabelas detalhadas podem ser consultadas em Oliveira (2005). 
transições constituem informações de grande relevância para políticas públicas de educação, qualificação, geração de emprego, políticas habitacionais e de saúde, pois saber que é positiva a permanência na escola como meio tanto de acumular capital humano quanto de adiar a transição para o processo de formação de família é de fundamental importância na redução de vários riscos aos quais os jovens estão expostos.

\section{Referências bibliográficas}

ALLISON, P. D. Survival analysis using SAS: a practical guide. 6. ed. Cary: SAS Institute, 2003.

BECKER, G. S. Human capital: a theoretical and empirical analysis, with special reference to education. New York: Columbia University, 1964.

BIDDECOM, A. E.; BAKILANA, A. Transitions into sex, parenthood and unions among adolescents and young adults in South Africa. (Paper presented at the Population Association of America, PAA Annual Meeting). Minneapolis, 2003.

BILLARI, F. C. et al. Household and union formation in a mediterranean fashion: Italy and Spain. Padova: Università di Padova: Max Planck Institute for Demographic Research, Rostock. Dipartimento di Scienze Statistiche, 2000.

BRASIL. Presidência da República. Trabalho infantil no Brasil: questões e políticas. Brasília: Presidência da República, 1998. Disponível em: <http://64.233.161.104/ search?q=cache:ZGOLOUNJ6tQJ:https:// www.planalto.gov.br/publi 04/COLECAO/ TRABIN.HTM +\%22Trabalho + infantil + no+ Brasil: +quest\%C3\%B5es +e + pol\%C3\% ADticas $\% 22 \& \mathrm{hl}=\mathrm{pt}-\mathrm{BR} \% 20$ target $=\mathrm{nw}>$.

CAMARANO. A. A. et al. A transição para a vida adulta: novos ou velhos desafios? Boletim Mercado de Trabalho - Conjuntura e Análise, Rio de Janeiro, n.21, p.53-66, fev. 2003.

CAVALLI, A.; GALLAND, O. (Eds.). Senza fretta di crescere. Napoli: Liguori, 1993.
Além das políticas públicas, informações dessa natureza são importantes para o planejamento estratégico da oferta de diversos serviços pelo setor privado. Dependendo das características sociodemográficas do universo investigado, podese delinear um mercado potencial para um leque de serviços que variam desde orientação vocacional até produtos e serviços para o ciclo de vida e familiar.

COPPOLA, L. Schooling and union formation as simultaneous processes: a comparative study of Italy and Spain. (Paper presented at the ESPE Conference). Bilbao, 2002.

COPPOLA, L.; AASSVE, A. Union formation and labour force participation in Italy and Spain: reciprocal causal relations and common determinants. Rome: Istat - Italian National Institute, 2003.

CORSEUIL, C.; SANTOS, D. D.; FOGUEL, M. N. Decisões críticas em idades críticas: a escolha de jovens entre estudo e trabalho em seis países da América Latina. ENCONTRO NACIONAL DE ESTUDOS POPULACIONAIS, 12, Caxambu, 2000. Anais... Belo Horizonte: ABEP, 2000 (Disponível em CDROM).

DEDECCA, C. S. Conceitos e estatísticas básicas sobre mercado de trabalho. In: OLIVEIRA, M. A. (Org.). Economia \& trabalho: textos básicos, Campinas, Unicamp/IE, v.1, p.95-112, 1998.

HAUSMANN, R.; SZÉKELY, M. Inequality and the family in Latin America. In: BIRDSAL, N.; KELLEY, A. C.; SINDING, S. W. (Eds.). Population matters: demographic change, economic and poverty in the developing world. New York: Oxford University, 2001. Cap.10, p.260-295.

IEDEMA, J.; BECKER, H.A.; SANDERS, K. Transitions into independence: a comparison of cohorts born since 1930 in the Netherlands. European Sociological Review, Philadelphia, v.13, n.2, p.117-137, 1997. 
KASSOUF, A. L. O efeito do trabalho infantil para os rendimentos dos jovens, controlando o background familiar. ENCONTRO NACIONAL DE ESTUDOS POPULACIONAIS, 13, Ouro Preto, 2002. Anais... Belo Horizonte: ABEP, 2002 (Disponível em CD$\mathrm{ROM})$.

. Trabalho infantil: escolaridade $x$ emprego. ENCONTRO NACIONAL DE ECONOMIA, 28. Anais... Campinas: Anpec, 2000 (Disponível em CD-ROM).

KLEIN, J. P.; MOESCHBERGER, M. L. Survival analysis: techniques for censored and truncated data. 2. ed. New York: Springer-Verlag, 2003.

LEME, M. C. S.; WAJNMAN, S. A alocação do tempo dos adolescentes brasileiros entre o trabalho e a escola. ENCONTRO NACIONAL DE ESTUDOS POPULACIONAIS, 12, Caxambu, 2000. Anais... Belo Horizonte: ABEP, 2000 (Disponível em CD$\mathrm{ROM}$ ).

MODELL, J.; FURSTENBERG, F. F. Jr.; HERSHBERG, T. Social change and transitions to adulthood in historical perspective. Journal of Family History, v.1, n.1, p.7-32, 1976.

OLIVEIRA, E. L. Transições: três aplicações a partir de dados das pesquisas domiciliares no Brasil. Tese de doutorado em Demografia. Belo Horizonte: Cedeplar, 2005. Disponível em: <http:// www.cedeplar.ufmg.br/demografia/teses/ 2005/Elzira_Lucia_de_Oliveira.pdf $>$. Acesso em: $\overline{2} 2$ nov. 2005.

RETHERFORD, R. D.; CHOE, M. K. Statistical models for causal analysis. New York: Wiley-Interscience, 1993.

SHANNAHAN, M. J. Pathways to adulthood in changing societies: variability and mechanisms in life, 2000. Annual Review of Sociology, Palo Alto, v.26, n.1, p.667-692, Aug. 2000.

SHIZZEROTTO, A.; LUCCHINI, N. Transitions to adulthood during the twentieth century: a comparative analysis of Great Britain, Italy and Sweden. United Kingdon: University of Essex, 2002 (EPAG Working Papers, n.36). Disponível em: $<$ http://www.iser.essex.ac.uk/epag/pubs/>. Acesso em: 16 mar. 2004.

STUPP, P.; CÁCERES, J. The relationship between age at completion of schooling and age at first birth in El Salvador. In: INTERNATIONAL UNION FOR THE SCIENTIFIC STUDY OF POPULATION GENERAL CONFERENCE, 24, 2001, Salvador, BA. XXIV IUSSP General Conference, Salvador - Brazil. Paris: IUSSP, 2001 (Disponível em CD-ROM).

SAS Institute Inc. The SAS System for Windows. Cary, NC, USA: SAS Institute Inc., 2001.

\section{Abstract \\ Transitions of young people into the labor market, first birth and leaving school: the Brazilian case}

This article investigates the relationship between the ages when young people leave school, enter the labor market and set up their own families. The Survival Analysis methodology was used. A descriptive analysis was carried out through a non-parametric model (Kaplan Meier) to estimate survival functions for each of these transitions, by gender. A semi-parametric model (Cox's Proportional Risks Model) was also used, which included variables such as mother's and father's educational levels and situation of residence until age 15. The database used in this study was the Life Patterns Research. The results captured the experience of transition of persons ages 20 to 49 at the time of the study (1996-1997). They therefore do not necessarily represent the experience of young people who are in transition in this first decade of the 21st century. The findings of the study are important basically due to this characteristic. If, for the 
experience of these cohorts, there is clearly a sequence of transitions, and the age at first child lowers the risk of leaving school by only $0.9 \%$, this is because the transition to first child is not the main reason for dropping out of school. Therefore, the challenges, the ages and the intervening factors seen constitute very important information for public policies in education, training, generation of jobs, habitation and health.

Key words: Transitions of young people. Labor market. Survival analysis.

Recebido para publicação em 06/05/2005.

Aceito para publicação em 14/10/2005. 